\title{
Homeostatic Insulin Sensitivity Indices Is the Detection of Gestational Diabetes Mellitus
}

\author{
Shahnaz Hayat ${ }^{1}$, Fatema Jebunnesa ${ }^{2}$, Nasreen Rosy ${ }^{3}$, Liaquat Ali $^{2}$ \\ ${ }^{1}$ Department of Gynaecology and Obstetrics, Dhaka Medical College, Dhaka, Bangladesh \\ ${ }^{2}$ Department of Biochemistry and Cell Biology, Faculty of Sciences, Bangladesh University of Health Sciences, Dhaka, Bangladesh \\ ${ }^{3}$ Department of Gynaecology and Obstetrics, Sir Solimullah Medical College and Hospital, Dhaka, Bangladesh
}

Email address:

runabd2005@yahoo.com(F. Jebunnesa)

\section{To cite this article:}

Shahnaz Hayat, Fatema Jebunnesa, Nasreen Rosy, Liaquat Ali. Homeostatic Insulin Sensitivity Indices Is the Detection of Gestational Diabetes Mellitus. American Journal of Biomedical and Life Sciences. Vol. 7, No. 6, 2019, pp. 159-163.

doi: $10.11648 /$ j.ajbls.20190706.16

Received: September 23, 2019; Accepted: November 22, 2019; Published: December 2, 2019

\begin{abstract}
Background: Early identification of GDM is strongly warranted for prevention of both maternal and fetal complications, but well known disadvantages of the present methods based on oral glucose challenge reduces the compliance and applicability of these methods in the screening of the disorder. Aims: The study aimed to assess FBG-based insulin sensitivity indices (ISIs) regarding their suitability as alternatives of $2 \mathrm{hr} 75$-g OGTT. Methods and Materials: Out of 300 subjects, 112 had GDM. Finally 84 GDM and 82 normal mothers were analyzed. A nested case control study was conducted with group of pregnant mothers, at 24 to 32 weeks of gestation, were recruited from BIRDEM (the tertiary hospital of Diabetic Association of Bangladesh) was screened for GDM by adapting WHO criteria. Serum glucose and insulin was measured by glucose oxidase and chemluminescence based ELISA. (ISIs) as well as glycemic and insulinemic indices were calculated their ability to detect GDM. Homeostatic formulas were used to quantify insulin sensitivity and B-cell function. McNamara test was used to calculate sensitivity, specificity, PPV and NPV of various tests against the gold standard of OGTT. Results: HOMA\%B was significantly $(\mathrm{p}<0.001)$ lower in GDM $(113.3 \pm 51.4)$ than their non-GDM counterparts $(207.9 \pm 91.3)$. In Pearson's correlation, HOMA\%B had a significant correlation with age, FBG, 75-g OGTT and fasting insulin level. HOMA\%S showed significantly correlation with FBG, 75-g OGTT, fasting insulin, HOMA\%B and QUICKI. Logistic regression provided significant association of HOMA\%B with GDM ( $\mathrm{p}=0.002)$ after adjusting the effect of the confounders. The value of different screening markers for predicting GDM was explored. HOMA\%S at optimum cut-off value of 50 showed sensitivity of $50 \%$ and specificity of 56\%, with PPV and NPV 56\% and 55\% respectively. QUICKI had $28 \%$ and $31 \%$ respectively at an optimum cut-off value of 0.54 . Fasting insulin showed $54 \%$ and $49 \%$ respectively at cut-off value of $12.9 \mu \mathrm{U} / \mathrm{ml}$ with PPV $50 \%$ and NPV $50 \%$. At an optimum cut- off value of $5 \mathrm{mmol} / 1$, the sensitivity, specificity, PPV and NPV of FBG was $82 \%, 78 \%, 79 \%$ and $81 \%$ respectively The corresponding value for combined fasting glucose and fasting insulin were $84 \%, 79 \%, 82 \%$ and $82 \%$.Conclusion: The data suggest that (ISIs), such as simple fasting blood glucose with a cut-off value of $5.0 \mathrm{mmol} / 1$, for Bangladeshi population, seems to be an acceptable test in the detection of GDM.
\end{abstract}

Keywords: GDM, Insulin Indices, Insulin Secretory Capacity, Insulin Sensitivity

\section{Introduction}

Gestational diabetes mellitus (GDM), a state of varying degree of glucose intolerance with onset or first recognition during pregnancy, is important for its obstetrics repercussion. It causes increased risk of maternal and perinatal morbidity and mortality. GDM is the most common medical complication and metabolic disorder of pregnancy [1]. Prevalence of GDM is increasing worldwide with higher prevalence in South-Asian women. It complicates up to $14 \%$ of pregnancy depending upon population described and the criteria used for diagnosis. The well known complications of GDM not only increase the maternal and perinatal morbidity and mortality, also have long term deleterious effect on mothers and their children increasing economic burden of a 
country. So early identification of GDM is strongly warranted. But the quest for a definitive screening test for GDM still goes on. In search of a suitable screening test for GDM, several FBG- based insulin sensitivity indices have been tried and showed reasonable accuracy over OGTT in a pilot study abroad. But for the feasibility of performing mass screening it should undergo further evaluation in different populations. So the current study has been carried out to assess several insulin sensitivity indices in search of a definitive screening test for GDM which would be reliable, simple and patient friendly for Bangladeshi population.

\section{Methodology}

On receipt of the consent form, fasting and 2-hour after 75-g glucose, blood sample were collected from volunteers who met under the selection criteria of the study subjects. Detailed socio-demographic data, family history and medical history were recorded on a pre- designed data collection sheet appropriately. All interviews were conducted in the hospital. Physical examination was done and anthropometric measurements (height, weight) of each subject were taken and recorded in a pre designed data collection sheet. Obstetric examination was performed and recorded for every patient. The data and the specimen (blood) were collected in every morning at BIRDEM hospital.

Serum glucose was measured by glucose oxidase method and insulin was assayed with a chemluminescence based ELISA. Insulin sensitivity indices as well as glycemic and insulinemic indices were calculated and tested for their ability to detect GDM. Homeostatic formulas were used to quantify insulin sensitivity and B-cell function. Data were analyzed by appropriate statistical tests (using SPSS
Windows 11.0). McNamara test was used to calculate sensitivity, specificity, PPV and NPV.

\section{Results}

The GDM group had higher age as compared to control (years, $\mathrm{M} \pm \mathrm{SD}, 28.9 \pm 3.8$, vs. 26.7 $\pm 4.6, \mathrm{p}<0.001$ ). (Table1) HOMA $\%$ B was significantly $(\mathrm{p}<0.001)$ lower in GDM $(113.3 \pm 51.4)$ than their non-GDM counterparts $(207.9 \pm 91.3)$. (table2)QUICKI of GDM was $0.52 \pm .03$ and that of control was $0.55 \pm .05$; the difference was statistically significant $(\mathrm{p}<0.001)$. (Figure 1$) \mathrm{HOMA} \% \mathrm{~S}$ showed no significant difference ( $\mathrm{p}=0.158)$ between GDM and non-GDM groups. (Table2)In Pearson's correlation analysis HOMA\%B had a significant correlation with age, FBG, 75-g OGTT and fasting insulin level. HOMA\%S showed statistically significant correlation with FBG, 75-g OGTT, fasting insulin, HOMA\%B and QUICKI. Logistic regression analysis provided significant association of $\mathrm{HOMA} \% \mathrm{~B}$ with GDM ( $p=0.002$ ) after adjusting the effect of the confounders. (Table3)The value of different screening markers for predicting GDM was explored. HOMA\%S at optimum cutoff value of 50 showed sensitivity of $50 \%$ and specificity of $56 \%$, with PPV and NPV 56\% and 55\% respectively. (Table4)QUICKI had sensitivity and specificity of $28 \%$ and $31 \%$ respectively at an optimum cut-off value of 0.54 . Fasting insulin showed sensitivity and specificity of 54\% and $49 \%$ respectively at cut-off value of $12.9 \mu \mathrm{U} / \mathrm{ml}$ with PPV $50 \%$ and NPV 50\%.At an optimum cut- off value of $5 \mathrm{mmol} / 1$, the sensitivity, specificity, PPV and NPV of FBG was $82 \%, 78 \%, 79 \%$ and $81 \%$ respectively The corresponding value for combined fasting glucose and fasting insulin were $84 \%, 79 \%, 82 \%$ and $82 \%$.

Table 1. Clinical characteristics of the study subjects.

\begin{tabular}{llll}
\hline Variable & Control $(\mathbf{n}=\mathbf{8 2})$ & GDM $(\mathbf{n}=\mathbf{8 4})$ & P Value \\
\hline Age $(\mathrm{yrs})$ & $26.7 \pm 4.6$ & $28.9 \pm 3.8$ & $<0.001$ \\
Gestational week & $26 \pm 8.9$ & $24.9 \pm 8.3$ & 0.448 \\
Parity & $2(1-7)$ & $1(1-5)$ & 0.883 \\
BMI $\left(\mathrm{Kg} / \mathrm{m}^{2}\right)$ & $26.1 \pm 4$ & $27.2 \pm 4$ & 0.056 \\
SBP $(\mathrm{mm}$ of $\mathrm{Hg})$ & $110.8 \pm 10.84$ & $113.9 \pm 12.6$ & 0.634 \\
DBP $(\mathrm{mm}$ of $\mathrm{Hg})$ & $74.3 \pm 7.9$ & $74.9 \pm 8.7$ & 0.770 \\
\hline
\end{tabular}

$\mathrm{GDM}=$ Gestational diabetes mellitus; $\mathrm{BMI}=$ Body Mass Index; $\mathrm{SBP}=$ Systolic blood pressure; $\mathrm{DBP}=\mathrm{Diastolic}$ blood pressure.

Table 2. Glycemic and Insulinemic status of the study subjects.

\begin{tabular}{llll}
\hline Variable & Control $(\mathbf{n = 8 2})$ & GDM $(\mathbf{n = 8 4})$ & P Value \\
\hline Fasting blood glucose $(\mathrm{mmol} / \mathrm{l})$ & $4.5 \pm 0.73$ & $6.37 \pm 1.6$ & $<0.001$ \\
75 gm OGTT & $6.62 \pm 6.7$ & $11.0 \pm 3$ & $<0.001$ \\
Fasting insulin (microU/ml) & $15.96 \pm 9$ & $13.6 \pm 3.2$ & 0.039 \\
Fasting insulin (picomole/l) & $110.8 \pm 63.1$ & $94.4 \pm 22.1$ & $<0.001$ \\
HOMA\%B & $207.9 \pm 91.3$ & $113.3 \pm 51.4$ & $<0.001$ \\
HOMA\%S & $50.9 \pm 15.9$ & $48.6 \pm 10.1$ & 0.158 \\
GIR & $0.34 \pm 0.11$ & $0.49 \pm 0.1$ & $<0.001$ \\
QUICKI & $0.55 \pm 0.05$ & $0.52 \pm 0.03$ & $<0.001$ \\
\hline
\end{tabular}

$\mathrm{GDM}=\mathrm{Gestational}$ diabetes mellitus; OGTT=Oral Glucose Tolerance; HOMA $\% \mathrm{~B}=$ Homeostasis Model Assessment of $\beta$ cell capacity; HOMA $\%$ S = Homeostasis Model Assessment of Insulin Sensitivity; GIR=Glucose Insulin Ratio; QUICKI = Quantitative Insulin Sensitivity Check Index 
Table 3. Correlation of HOMA\%B and $H O M A \% S$ with other variables of the study subjects.

\begin{tabular}{|c|c|c|c|c|}
\hline \multirow{2}{*}{ Variable } & \multicolumn{2}{|c|}{ HOMA\%B all subjects $(n=166)$} & \multicolumn{2}{|c|}{ HOMA\%S all subjects $(n=166)$} \\
\hline & $\mathbf{r}$ & p & $\mathbf{r}$ & p \\
\hline Age (yrs) & -0.168 & 0.030 & -0.078 & 0.319 \\
\hline Fasting blood glucose $(\mathrm{mmol} / \mathrm{l})$ & -0.688 & $<0.001$ & -0.212 & 0.006 \\
\hline 75 gm OGTT & -0.524 & $<0.001$ & -0.155 & 0.044 \\
\hline Fasting insulin (microU/ml) & 0.682 & $<0.001$ & -0.836 & $<0.001$ \\
\hline НОМА $\%$ В & - & - & -0.369 & $<0.001$ \\
\hline HOMA\%S & -0.369 & $<0.001$ & - & - \\
\hline GIR & -0.821 & $<0.001$ & 0.452 & $<0.001$ \\
\hline
\end{tabular}

HOMA $\%$ B =Homeostasis Model Assessment of $\beta$ cell capacity; HOMA\%S = Homeostasis Model Assessment of Insulin Sensitivity; GIR=Glucose Insulin Ratio; QUICKI = Quantitative Insulin Sensitivity check Index

Table 4. Predictive values of different marker in the study subjects $(n=166)$.

\begin{tabular}{|c|c|c|c|c|c|c|}
\hline Variable & Percentile & Cut-off Value & Sensitivity (\%) & Specificity (\%) & PPV(\%) & NPV(\%) \\
\hline \multirow{3}{*}{$\begin{array}{l}\text { Fasting Glucose and } \\
\text { Fasting Insulin }\end{array}$} & $25^{\text {th }}$ & & $1 \%$ & $2 \%$ & $61 \%$ & $1 \%$ \\
\hline & $50^{\text {th }}$ & & $84 \%$ & $79 \%$ & $82 \%$ & $82 \%$ \\
\hline & $75^{\text {th }}$ & & $27 \%$ & $1 \%$ & $1 \%$ & $66 \%$ \\
\hline \multirow{3}{*}{ НОМА\%B } & $25^{\text {th }}$ & 104.7 & $54 \%$ & $1 \%$ & $36 \%$ & $2 \%$ \\
\hline & $50^{\text {th }}$ & 138.9 & $22 \%$ & $23 \%$ & $23 \%$ & $22 \%$ \\
\hline & $75^{\text {th }}$ & 203 & $71 \%$ & $56 \%$ & $14 \%$ & $36 \%$ \\
\hline \multirow{4}{*}{ HOMA\%S } & $15^{\text {th }}$ & 37 & $13 \%$ & $82 \%$ & $44 \%$ & $48 \%$ \\
\hline & $25^{\text {th }}$ & 42 & $29 \%$ & $79 \%$ & $59 \%$ & $52 \%$ \\
\hline & $30^{\text {th }}$ & 44 & $36 \%$ & $76 \%$ & $62 \%$ & $54 \%$ \\
\hline & $50^{\text {th }}$ & 50 & $55 \%$ & $56 \%$ & $56 \%$ & $55 \%$ \\
\hline \multirow{3}{*}{ QUICKI } & $25^{\text {th }}$ & 0.50 & $73 \%$ & $10 \%$ & $45 \%$ & $29 \%$ \\
\hline & $50^{\text {th }}$ & 0.54 & $28 \%$ & $31 \%$ & $3 \%$ & $30 \%$ \\
\hline & $75^{\text {th }}$ & 0.57 & $10 \%$ & $58 \%$ & $20 \%$ & $39 \%$ \\
\hline
\end{tabular}

$\mathrm{PPV},=$ Positive predictive value; NPV= Negative predictive Value, HOMA $\% \mathrm{~S}=$ Homeostasis Model Assessment of Insulin Sensitivity; GIR $=\mathrm{Glucose}$ Insulin Ratio; QUICKI=Quantative Insulin Sensitivity check Index

\section{Discussion}

Today the majority of women with GDM have features of type 2 diabetes, and are older, more obese and of higher parity [2]. In the present study we analyzed age, parity and BMI by a case-control comparison. Only age of the patient was found to be different between GDM and non-GDM groups. On average GDM mothers were 2.26 years older than controls $(\mathrm{p}<.001)$.

The HOMA-S is closely correlated with ISI assessed by the euglycemic clamp method which is regarded as the 'gold standard' for insulin resistance[3]. However, euglycemic clamp is complex, requires multiple blood sampling and is inconvenience in pregnancy[4]. A criticism of the HOMA-S is its deviation from linearity with increasing insulin resistance; consequently, it is believed to be an inaccurate index for those with advanced type 2 diabetes[5].Thus, the author claimed that HOMA-S provided a weaker predictive index compared to QUICKI which is based on a logarithmic and reciprocal transformation of a single fasting glucose and insulin value [5].The model is very similar to HOMA and differs only in the treatment of the data. This has been validated against the isoglycemic-hyperinsulinemic clamp and was found to have a good linear correlation $(\mathrm{r} 2=$ 0.61)[4]. Measuring insulin sensitivity by a fasting method (like HOMA\%S or QUICKI) is not only simpler but also noninvasive requiring only a single venipuncture. It is cheaper, less labor-intensive and less time consuming [6].In our study, for measuring insulin resistance among GDM and non-GDM, HOMA-S and QUICKI were compared by using sensitivity and specificity calculations based on insulin resistance with ROC analysis. Among these ISIs HOMA\%S shows more sensitivity and specificity followed by QUICKI. The 50th percentile of cut-off value (54) was found to be optimum where the sensitivity of $\mathrm{HOMA} \% \mathrm{~S}$ was $55 \%$ and PPV 56\% (Table 4). For QUICKI the maximum sensitivity and PPV were found at 25 th percentile cut-off value $(45 \%$ and 29\% respectively) (Table 4). These findings are reflected in a study by [6] on comparing HOMA, GIR and QUICKI to measure insulin sensitivity. But Katz et al [5] contradicted us by suggesting QUICKI as a novel, accurate and reproducible method for determining insulin sensitivity. Kirwan et al [4] also demonstrated HOMA as a weaker predictive index compared with QUICKI. Though area under curve (AUCs) that were derived from the ROC curves for HOMA $\% \mathrm{~S}$ and QUICKI were statistically significant, the sensitivity and predictivity of HOMA\%S and QUICKI are not high (Table 4). Since the failure to detect $44 \%$ of cases of GDM by HOMA $\%$ S and $55 \%$ of cases by QUICKI, they leave a large number false negative case and may not be considered as 
reliable tests in the detection of GDM. Our observation is not reflected by finding of Kauffman [7] who revealed comparable sensitivity and specificity of HOMA $(68 \%$ and $74.5 \%)$ and QUICKI (87.5\% and 57\%) with others common screening tests.

A study by Buchanan et al [8] suggested that $\beta$ - cell dysfunction is a common, if not universal, feature of GDM. The HOMA\% B is the calculation based on fasting glucose and insulin concentrations to percent B-cell function using a mathematical model. This test has been well correlated with insulin-mediated glucose disposal assessed by the glucose clamp technique[3]. In our study, the GDM cases exhibit the significant defect in secretory capacity of beta cell (Table 2). From ROC curve analysis HOMA\%B shows more sensitivity and specificity than ISIs (Table4). In fact there is evidence in favor of a predominant role of beta cell dysfunction in the genesis of type 2 diabetes in Bangladeshi population[9].Xiang et al [10] also found that GDM had a $67 \%$ reduction in their beta cell compensation compared with normal pregnant control subjects. Kauffman et al [7] argued against the use of $\mathrm{HOMA} \% \mathrm{~B}$ independently as screening tools. Because it is impossible to ascertain whether increased level of insulin is in response to mounting insulin resistance or decreased level due to B-cell dysfunction. However HOMA $\% \mathrm{~B}$ has been claimed to be more suitable for large epidemiological studies [3].

When none of the ISIs, HOMA\%B or fasting insulin was found to show a reasonably high level of sensitivity, specificity, PPV or NPV, an analysis was made with FBG as a marker. The AUC (0.883) derived from the ROC curve for FBG was highly significant and it was found to be the best predictor of GDM. The best cut-off value from the ROC curve was found to be $5.0 \mathrm{mmol} / 1$ and with this cut-off point the sensitivity and specificity of this simple parameter were $82 \%$ and $78 \%$ respectively, and the PPV and NPV were $79 \%$ and $81 \%$ (Table 4). This finding strongly corresponds with observation by Kauffman [7]. They demonstrated FBG as the single most discriminatory test at cut-off value of $92 \mathrm{mg} / \mathrm{dl}$ (5.01 mmol/l) (sensitivity $76 \%$ \& specificity $89.8 \%$ ) for the diagnosis of GDM. In another study, Perucchini [11] also showed FBG (using a cut-off value of $4.8 \mathrm{mmol} / 1$ with sensitivity of $81 \%$ specificity of $76 \%$ ) as an easier means of screening for GDM than glucose based test. There is disagreement with Kausta et al [12] who found that a single fasting glucose screen failed to identify $60 \%$ of women with abnormal 2 hour blood glucose levels. There are several other studies [13,11,14-16] concluded that FBG demonstrates equal or better sensitivity and specificity to screen for GDM than 50g OGTT which is recently recommended as universal screening $[7,17,18]$. Metzger et al [19] showed that a 1-hour $50 \mathrm{~g}$ OGTT would have $80 \%$ sensitivity at cut-off value of $140 \mathrm{mg} / \mathrm{dl}$ and the sensitivity can be increased to $90 \%$ further decreasing the threshold to $130 \mathrm{mg} / \mathrm{dl}$. This cut-off value will increase the number of women who require a 3-hour $100 \mathrm{~g}$ OGTT $25 \%$ and consequently will increase the cost of identifying each case of GDM. Again some authors [11,20] found that $50 \mathrm{~g}$ OGTT had relatively poor sensitivity.
Kauffman et al [7] supported that feasibility of FBG as a screen with great consideration, though women with postprandial hyperglycemia and fasting normoglycemia would be missed. The screening value of FBG can only be marginally improved by combining it with fasting insulin (sensitivity $84 \%$, specificity $79 \%$, PPV $82 \%$ and NPV $82 \%$ ) (Table 4). Combining the substantial cost of insulin assay this improvement in PPV and NPV may not be justified as a routine procedure.

\section{Conclusion}

The study results suggest that the insulin sensitivity indices do not seem to be reliable alternatives for the detection of GDM. Rather simple fasting blood glucose (with a cut-off value of $5.0 \mathrm{mmol} / \mathrm{L}$ for Bangladeshi population.

\section{Acknowledgements}

We are grateful to the staff of BUHS, BIRDEM and all the field staff who actively helped in the data collection and verification by all visits.

\section{References}

[1] Janzen C, Greenspoon JS, Palmer SM. Diabetes mellitus and pregnancy. Current Obstetric Gynecologic Diagnosis Treatment. 2003: 326-38.

[2] Edmond, D. K. (ed.), Dewhurst's Text book of Obstetrics and Gynecology, $7^{\text {th }}$ edn., Oxford University Press, London, 2007.

[3] Matthews, D. R., Hosker, J. P., Rudenski, A. S. et al., 'Homeostasis model assessment, insulin resistance and B-cell function from fasting glucose and insulin concentration in man', Diabetes, vol. 28, pp. 412-419, 1985.

[4] Kirwan, J. P., Huston-Presley, L., Kalhan, S. et al., 'Clinically useful estimates of insulin sensitivity during pregnancy: validation studies in women with normal glucose tolerance and gestational diabetes mellitus'.Diabetes Care, vol. 24, pp. 1519-21, 2001.

[5] Katz, A., Nambi, S. S., Mather, K. et al., 'Quantitative insulin sensitivity check index: a simple, accurate method for assessing insulin sensitivity in humans'.J Clin Endocrinol Metabl,vol. 85, pp. 2402-2410, 2000.

[6] M Keskin; S Kurtoglu; M Kendirci; M. Emre Atabek and C Yazici 'Homeostasis Model Assessment Is More Reliable Than the Fasting Glucose/Insulin Ratio andquantitative Insulin Sensitivity Check Index for Assessing Insulin Resistance Among Obese Children and Adolescents'. American Academy of Pediatrics. Vol. 115 No. 4 April 2005.

[7] Kauffman, R. P., Castracane, V. D., Peghee, D. et al., 'Detection of gestational diabetes mellitus by homeostatic indices of insulin sensitivity: A preliminary study', $\mathrm{Am} \mathrm{J}$ Obstet Gynecol, vol. 194, pp. 1576-1584, 2006.

[8] Buchanan, T. A., 'Pancreatic B-cell defects in gestational diabetes: implication for the pathogenesis and prevention of type 2 diabetes'. JCEM, vol. 86, no. 3, pp. 989-993, 2001. 
[9] M N Roy, K B Biswas, N Siddiqua, L Ali. 'Determinants of Insulin Secretion and Sensitivity in Bangladeshi Type 2 Diabetic Subjects'. Metabolic syndrome and related disorders 5 (3): 275-8, 2007.

[10] Xiang, A. H., Peters, R. K., Trigo, E. et al., 'Multiple metabolic defects during late pregnancy in women with high risk for type 2 diabetes mellitus', Diabetes Care, vol. 48, pp. 848-854, 2001.

[11] Perucchini, D., Fischer,U., Spinal, G. A. et al, 'Using fasting plasma glucose concentrations to screen for gestational diabetes mellitus: prospective population based study. $B M J$, vol. 319, pp. 812-815, 1999.

[12] Kousta, E., Laurence, N. J., Penny, A. et al., 'Implication of new diagnostic criteria for abnormal glucose homeostasis in women with previous gestational diabetes', Diabetes Care, vol. 22, pp. 933-937, 1999.

[13] Rey, E., Hudon, L., Michon, N. et al, 'Fasting plasma glucose versus glucose challenge test: screening for gestational diabetes and cost effectiveness', Clin Biochem, vol. 37, pp. 780-784, 2004.

[14] Mortensen, H. B., Molsted-Pedersen, L., Kuhl, C. et al.1999, 'Screening procedure for diabetes in pregnancy', Diabetes Metab, vol. 11, pp. 249-253.
[15] Reichelt, A.J., Spichler, E.R., Branchtein, L. et al., 'Fasting plasma glucose is a useful test for the detection of gestational diabetes: Brazilian study of gestational diabetes working group (EBDG) ', Diabetes Care, vol. 21, pp. 12461249,1998

[16] Sacks, D.A., Greenspoon, J.S. \& Fotheringhan, N. 'Could the fasting plasma glucose assay be used to screen for gestational diabetes?' J Reprod Med, vol. 37, pp. 907-9, 1992.

[17] American College of Obstetricians and Gynecologists Committee on Practice Bulletin, 'Clinical management guidelines for obstetrician-gynecologists: gestational diabetes', Am JObstet Gynecol, vol. 98, no 30, pp. 525-538, 2001.

[18] American Diabetes Association, 'Report of the expert committee on the diagnosis and classification of diabetes mellitus', Diabetes Care, vol. 21, pp. 1183-1187, 1997.

[19] Metzger, B. E. \& Coustan, D. M., 'Organizing committee: summary and recommendations of the fourth international workshop conference on gestational diabetes mellitus', Diabetes Care vol. 21, no.suppl.2, pp. B161-B167, 1998.

[20] Van Turnhout, H. E., Lotgering, F. K. \& Wallenburg, H. C. 'Poor sensitivity of the fifty gram one-hour glucose screening test for hyperglycemia', Eur J Obstet Gynecol Repord Biol, vol. 53, pp. 7-10, 1994. 